\title{
An Adaptive BP Neural Network Model for Teaching Quality Evaluation in Colleges and Universities
}

\author{
Yong Jin $\mathbb{D}^{1},{ }^{1}$ Yiwen Yang, ${ }^{2}$ Baican Yang, ${ }^{2}$ and Yunfu Zhang ${ }^{3}$ \\ ${ }^{1}$ Department of Academic Affairs, Shanghai University of Traditional Chinese Medicine, Shanghai 201203, China \\ ${ }^{2}$ Department of Pharmacy, Shanghai University of Traditional Chinese Medicine, Shanghai 201203, China \\ ${ }^{3}$ Department of Organization, Yunnan University of Traditional Chinese Medicine, Kunming 650000, China
}

Correspondence should be addressed to Yong Jin; jinyong@shutcm.edu.cn

Received 20 June 2021; Revised 17 July 2021; Accepted 26 July 2021; Published 15 August 2021

Academic Editor: Shan Zhong

Copyright ( 2021 Yong Jin et al. This is an open access article distributed under the Creative Commons Attribution License, which permits unrestricted use, distribution, and reproduction in any medium, provided the original work is properly cited.

There is currently no fair, rational, or scientific approach for evaluating college teachers' teaching abilities. Mathematical methods are frequently used to measure the teaching capacity of college instructors in order to make it more scientific. Traditional statistical analysis evaluation models, fuzzy evaluation methods, grey decision methods, and the analytic hierarchy process (AHP) are only a few examples. Because teacher assessment is a nonlinear problem, even though the preceding methods have produced some positive results, they are vulnerable to some subjectivity. In this paper, the neural network model is incorporated into the adaptive vector and momentum of the modified BP neural network of a gradient descent method to boost the model's convergence speed, and the model is thoroughly researched to evaluate university teaching quality, and the network structure is omitted to address the complex nonlinear problem of college and university teaching quality assessment. The model's comprehensive evaluation of teaching activities is then bolstered by the addition of new evaluation indexes to the existing ones.

\section{Introduction}

With the rapid development of higher education [1-4], a school's reputation has become the most important criterion for students when choosing a school, and a school's reputation is largely determined by the quality of its education [5-7]. A school's top priority is education quality, because it has an impact on not only the school's existence and development but also the future and destiny of its students. However, as a result of the large-scale enrollment increase of schools and universities for several years in a row, a number of related issues have emerged, including a teacher shortage, a decline in the quality of students, and a shortage of educational and teaching equipment and logistics facilities. These issues have sparked widespread concern in society, prompting a discussion about how to address them. Education quality has become a contentious issue, but it has also evolved into a comprehensive reflection of college and university work.

Improving educational quality is always a hot topic in higher education. The idea of "improving higher education quality" is very practical. As a result, the internal assessment activity places a strong emphasis on the creation of a system for evaluating teaching quality. The teaching quality of colleges and universities is reflected in the teaching quality of each specialty or department, the teaching quality of each department is reflected in the quality of each course, and the course quality is reflected in the teaching quality of each teacher who teaches the course $[8,9]$. The quality and degree of teachers have an impact on how a school is run. The quality and level of teachers determine the administrative direction of a school, the quality of education and instruction, and the impact of educational reform.

Teaching is the most important aspect of schoolwork. The effectiveness of a school's teaching is a key determinant of its success [10]. Teaching quality management is critical to the school's overall quality management. Evaluation of teachers' teaching quality aids school leaders and administrators in determining the degree to which teaching goals have been met, grasping the school's teaching work comprehensively and accurately, and improving teaching quality. The topic of teaching quality evaluation [11-13] will also be 
discussed. This is a critical assignment. The cornerstone of teaching activities in colleges and universities, as well as the most significant connection in assuring the quality of talent development in these institutions, is teaching quality evaluation. As a result, assessing teaching quality is a critical component of promoting high-quality education, changing teaching, and improving teaching quality. It has a substantial impact on overall educational quality. Because teaching is both a spiritual labor and an art, there is no established formula. Nonquantitative criteria are widely utilized in the evaluation of teaching quality, because defining what constitutes teaching is very ambiguous and difficult to measure. The difficulty and complication of assessing quality teaching and learning are both part of the teaching process. It is far more difficult to evaluate the quality of a teacher's instruction than it is to evaluate the quality of a product. The teaching process is made up of a variety of components, and it is a two-way activity that involves both teachers and students. There are many factors that influence teaching quality, and there is still more to investigate. The development of a scientific and reasonable teaching quality evaluation system [14] that can accurately and equitably assess teaching quality is a significant challenge.

Because it is a complex and abstract nonlinear problem, it is difficult to express the evaluation of teaching quality at colleges and universities using a mathematical model or analytical formula. The neural network model [15-17] can achieve mutual mapping between any dimensions and has strong nonlinear processing capabilities [18, 19]. As a result, creating a neural network model to handle the challenge of evaluating the quality of college instruction is an effective metric. Computer vision [20-22] in the evaluation of teaching quality in colleges and universities not only aids in improving teaching quality, promoting continuous improvement of teaching goals, and promoting scientific education decision-making, but it also aids in the development of intelligent and standardized teaching management in colleges and universities.

The following are the main innovations points of this paper:

(1) This study presents a novel adaptive BP neural network model that can adequately evaluate the teaching quality and teaching effect of colleges and universities in order to properly evaluate the teaching quality and teaching effect of colleges and universities

(2) As a novel adaptive BP neural network, a model is proposed in this study. The model contains an adjustable learning rate and momentum term to improve the gradient descent method of the BP neural network's convergence speed and optimize the network topology to ensure the model's stability

\section{Background}

Teaching quality evaluation in college is a complicated topic that encompasses variables such as teaching conditions, class difficulty, instructor teaching and learning effect of many aspects, such as their interaction, and the complex relationship between teachers and students at the same time; factors affecting the quality of teaching are also more; at present, none of them has been recognized; the ideal teaching quality evaluation system, in the context of existing research status, focuses on three aspects: the first is the study of the evaluation subject, the second is an investigation into the content of the teaching quality assessment system, and the third is an investigation on how to evaluate the teaching quality grading technique once each index in the system has been determined.

Teachers' self-evaluation, peer evaluation [23], administrative leadership evaluation, expert assessment, and student evaluations of teachers are all examples of ways or techniques to assess teaching quality. Because each evaluation method and its outcomes serve a different purpose in the evaluation, their evaluation functions should be distinct as well. Each evaluation method and its results are only a part of the overall assessment of teaching quality, but they cannot be compared. Because of the large number of college and university teachers, as well as the frequent evaluation of the number of teachers, the organization's leadership and peer expert survey evaluation method is not only timeconsuming. Because of the interpersonal contact and lack of familiarity with the teaching process, as well as the influence of such aspects as actually difficult to operate, most colleges and universities use students as the major body of teachers' teaching quality evaluation technique. China's colleges and universities have used student evaluation of instructional activities since the 1980s, which has helped to improve teaching quality. Because they are the direct recipients of instruction, students have the right and ability to evaluate teachers' teaching. Because of the variety of types, complex majors, and uneven levels of students, different requirements for instructors' teaching quality evaluation exist in colleges and universities across the country.

On the content of the teaching level evaluation system design, it is difficult to take a course and a teacher in a learning phase, to quantify the effect of general courses as the main index, or teaching effect as the main index, and put the evaluation content on the teaching process, because learning and development is a continuous process and learning and growth environment is diverse. From the perspective of process management, the school teaching process is manifested as the interaction of multiple factors and the combination of multiple links; it is also difficult to compare different fields' teaching, different types of courses, teaching relationships, and teaching objects. As a result, the most basic features that can immediately represent the teaching level and are prevalent are mostly taken into account when evaluating teaching quality.

\section{Methodology}

3.1. Teaching Quality Evaluation System. The goal of teaching quality assessment is to promote teaching reform, improve teaching quality, reduce student burden, increase students' intelligence, and improve their ability to analyze and solve problems. We should be objective, fair, and rational 
when evaluating the quality of instruction, rather than guessing or mixing personal feelings, and we must achieve the unity of ideology, science, and feasibility. Teaching quality is frequently assessed in colleges and universities through four channels: student evaluation, expert evaluation, peer evaluation, and instructor self-assessment, with the final evaluation results synthesized. However, various concerns remain in the process of establishing, employing, and evaluating the teaching quality assessment system's evaluation outcomes, such as evaluation theory research, evaluation method use, evaluation method updates, and evaluation data analysis. These issues have an immediate impact on the educational system. The importance of quality assurance and the potential for knowledge mining.

The various indicators in the evaluation system generally involve teaching attitudes, teaching content proficiency, and basic teaching skills. However, the comprehensive quality of teachers is not only reflected in the above aspects but should also include teachers' knowledge, teaching and research ability, teaching design ability, and teacher's innovation ability, but these evaluation indicators that can fully reflect the comprehensive quality and personality of teachers are rarely involved in the evaluation system. As a result, the design of an evaluation index system should be given careful thought.

The indications in the evaluation system of the differences in the influence degree of the evaluation findings should be given distinct weights; however, many schools and universities still use the same weighting approach to make things easier for themselves or subjectively determined a weight distribution table to establish the evaluation system; using this evaluation approach to assess teaching quality not only undermines the credibility of evaluation outcomes but also creates barriers to further data mining. As a result, a suitable weight distribution is a critical step in perfecting the evaluation system.

The data in the teaching evaluation database is continuously growing as network teaching assessment becomes more prevalent on digital campuses. Facing the "mountain of data sets," traditional data analysis means can only obtain the surface information of these data, and it is difficult to dig out the deep valuable information. An essential goal of teaching evaluation is to learn how to better use evaluation data to extract knowledge. The lack of modern scientific and technology methodologies and an imperfect evaluation feedback and control mechanism are the major issues that the teaching quality evaluation system is currently experiencing. The correctness and trustworthiness of evaluation outcomes will be directly affected by the scientific establishment of assessment indexes and the rationality of index weight distribution. As a result, developing and establishing a method for evaluating teaching quality are a vital step in enhancing teacher quality and a pressing requirement in colleges and universities to improve classroom management.

Teachers' teaching quality is evaluated in terms of content, techniques, attitude, and teaching effect, such as content, in order to demonstrate consistency, comprehensiveness, and effectiveness, and the principle of independence, incentive, and fault tolerance; this article set evaluation index is from 1 to $7: X_{1}, X_{2}, X_{3}, X_{4}, X_{5}, X_{6}$, and $X_{7}$, where $X_{1}$ represents the course progress, the depth and breadth of teaching, and the reasonable degree of learning burden; $X_{2}$ represents the degree of integration with practice and whether it can reflect modern scientific and technological achievements; $X_{3}$ represents the degree of clarity, hierarchy, and emphasis of the lecture; $X_{4}$ stands for vivid explanation, inspiring and inducing, and attractive, exemplified by examples, combining theory with practice; $X_{5}$ stands for instructing correct learning methods to cultivate students' analytical ability; $X_{6}$ represents the degree of careful preparation for lessons, skilled explanation, answering questions, and correcting homework; and $X_{7}$ stands for continuous improvement in teaching and educating people.

3.2. Adaptive BP Neural Network. Existing teaching quality evaluation methods and models in colleges and universities have flaws in processing small-scale low-dimensional data sets, such as difficulty determining the index weights of the analytic hierarchy process, subjectivity and randomness of the fuzzy synthesis method, and the support vector machine's grid search. When using a traditional BP neural network, the optimization approach is slow, the self-convergence is slow, and it is easy to fall into the local minimum. An adaptive BP neural network model is proposed in this study. Integrating an adaptive learning rate and a momentum component to speed up convergence, as well as tuning the network structure to ensure model stability, improves the gradient descent methodology of the BP neural network. New evaluation indicators are added to the model's input feature vector to ensure a complete assessment of teaching activities, and the evaluation sample data set is normalized to improve the model's computation performance. The evaluation sample data set's training and test portions are separated and entered into the model for training and verification. To ensure that the model presented in this chapter is useful in assessing teaching quality at colleges and universities, performance indicators such as MSE, prediction accuracy, and training time are used to compare it to other approaches and models.

Given the fact that assessing teaching quality is a multiobjective, multilevel, and sophisticated nonlinear problem and current college teaching quality assessment methods and models make determining weights difficult, subjectivity and randomness are too strong, and the conventional BP neural network has a slow convergence time, making it easy to fall into a local minimum. The model of an adaptive BP neural network is proposed. The basic idea behind this model is to use an adaptive learning rate and a momentum term to improve the convergence speed of a BP neural network's gradient descent method and optimize the network topology to ensure the model's stability. In addition, to create the teaching quality evaluation index system of cost text, new evaluation indexes were added to the traditional evaluation indexes, ensuring the model's comprehensive evaluation of teaching activities, and the evaluation index sample data set was normalized as the model's input feature vector, improving the model's calculation efficiency. 
3.2.1. Network Structure. Any nonlinear mapping of $n$ -dimensional to $m$-dimensional functions can be satisfied by a three-layer BP neural network. As a result, this research develops a three-layer BP neural network model for evaluating teaching quality, with one input layer, one hidden layer, and one output layer. The size of the input sample and the size of the output result, respectively, determine the number of neurons in the input and output layers. Selecting neurons in the buried layer, on the other hand, is more difficult and not well guided by theory. This research uses an empirical formula and multiple experiments to identify the number of neurons in the input and output layers. Using equation (1) and trial and error, we establish the appropriate number of neurons.

$$
n_{i}=\sqrt{n+m}+a
$$

where the number of neurons in the input layer is $n$, the number of neurons in the output layer is $m$, and $a$ is a constant between $[1,10]$.

3.2.2. Adaptive Learning Rate and Momentum. In a standard $\mathrm{BP}$ neural network, the learning rate, also known as the learning step size, is fixed. When the learning rate is too high, the network topology becomes unstable and oscillates. However, if the learning rate is too low, the network's convergence speed will be slow, making it impossible to ensure that the learning efficiency of the overall network structure is optimal in real-world applications. To increase convergence speed, the adaptive learning rate automatically adjusts the learning rate in response to changes in network error and corrects the weights and thresholds between the connection layers on a regular basis. Assuming the initial learning rate is $\mu(0)$, the $n$-th iteration of the model results in a network error of $E(n)$. When the learning rate changes, the equation is shown as follows:

$$
\mu(n)= \begin{cases}\beta \mu(n-1), & E(n)<E(n-1), \\ \gamma \mu(n-1), & E(n)>E(n-1), \\ \mu(n-1), & \text { else, }\end{cases}
$$

where $\beta=1.05$ and $\gamma=0.7$.

Adaptively modifying the learning rate throughout the error back propagation process can significantly improve convergence speed. However, only the adjustment of the gradient descent direction at the current time $t$ is considered, and the gradient direction before the time $t$ is not considered, which will cause turbulence in the training process and cause the model to become unstable and easily fall into a local minimum. The momentum term is inserted to rectify this inconsistency, and it alters the weight value to give a damping effect in the error back propagation process. The equation is shown in (3). Regarding equation (3) as a time series with $t$ as a variable $(0<t<N)$, equation (3) can be regarded as the first-order difference equation of $\Delta w(n)$, and its calculation equation is as follows:

$$
\begin{aligned}
& \Delta w(n)=\alpha \Delta w(n-1)-\mu \frac{\partial E(n)}{\partial w(n)}, \\
& \Delta w(n)=-\mu \sum_{t=0}^{n} \alpha^{n-t} \frac{\partial E(n)}{\partial \mathrm{w}(n)},
\end{aligned}
$$

where $\alpha$ is the momentum term $(0<\alpha<1), w$ is the weight, $\mu$ is the learning rate, and $E(n)$ is the error. Then, the weight adjustment equation of BP neural network is as follows:

$$
w(n+1)=w(n)-\mu(n) \sum_{\mathrm{t}=0}^{N} \alpha^{n-t} \frac{\partial E(n)}{\partial \mathrm{w}(n)}
$$

To ensure a more thorough evaluation of the teacher's teaching process, 23 second-level indicators were produced by merging two first-level indicators of preteaching preparation and the situation in the teaching process with standard evaluation indicators in this study. Normalize the evaluation sample data to reduce the difficulty of altering the weight and threshold due to the large change in the input value, which will increase the calculation efficiency of the BP neural network.

3.3. Evaluation of University Teaching Quality Based on the Adaptive BP Neural Network. Figure 1 shows the processing flow chart for the adaptive BP neural network model for teaching quality evaluation. To increase the model's convergence speed, the model adjusts the learning rate based on a comparison of the current error and the prior error and adds a momentum term to dampen the model to prevent oscillations and assure stability. At the same time, new input variables are introduced. To ensure a fuller model evaluation, the input features are included. From the normalized evaluation index sample number set, a training data set and a test data set are constructed, with the training data set being used to train the model. Adjust the number of hidden layer neurons in the network structure of the model during the training process, using the adaptive learning rate and momentum term to improve the gradient descent method of the BP neural network as the training function. The rate and momentum terms are finetuned, and the model was continuously trained iteratively to modify its own weights and thresholds until the evaluation result meets the set target accuracy or reaches the maximum number of iterations, then exits the training and obtains this time the best or final model. Finally, use the test data set to demonstrate that the proposed model has good evaluation prediction accuracy and benefits in evaluating teaching quality.

\section{Experiments and Results}

4.1. Experimental Setup. In the experiment, the network structure of the model is given as three layers, the learning rate is set to 0.001 , the maximum number of training times is 10000, the weights and thresholds of the model are randomly selected, and the activation function is a single sigmoid function. 


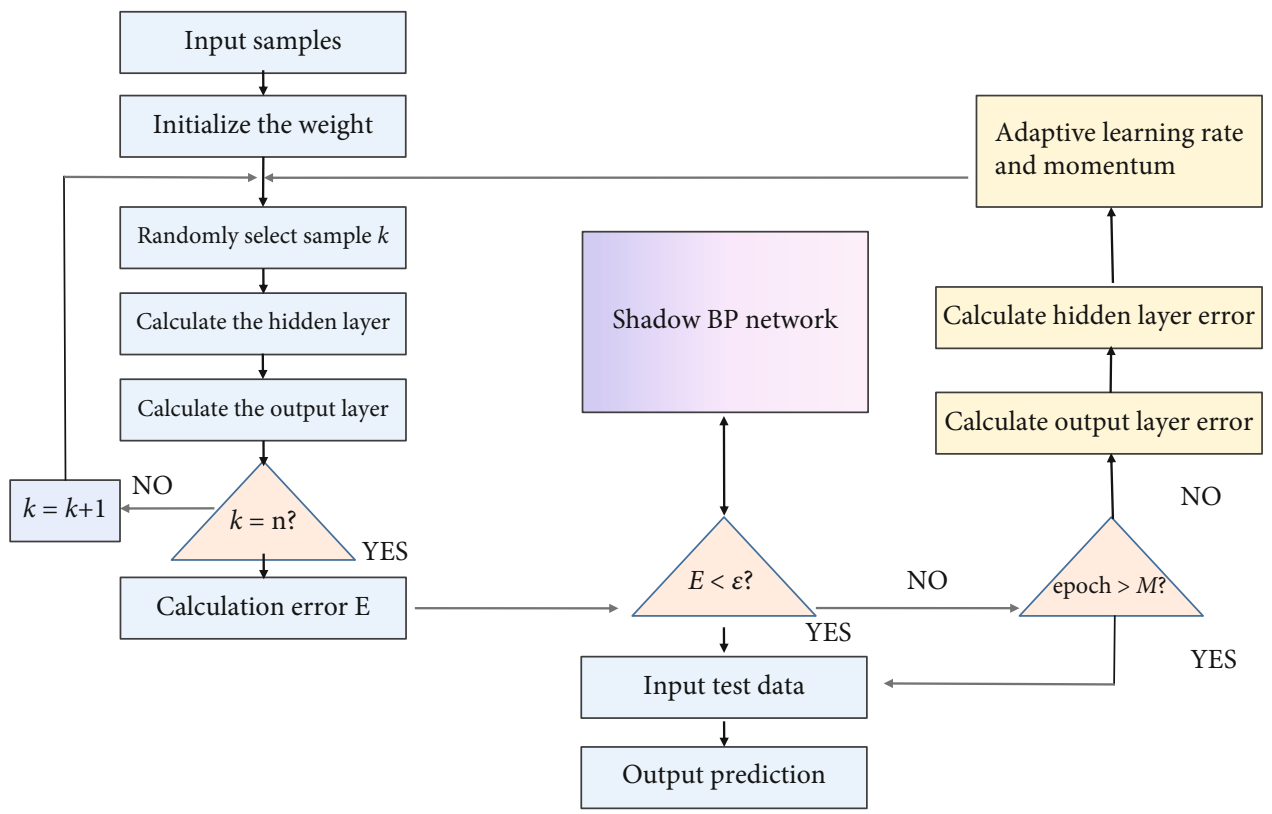

Figure 1: Evaluation model of teaching quality in colleges and universities.

4.2. Dataset. Obtain the evaluation data set of a course from 2003 to 2020 from the educational administration system of a university. There are a total of 7171 sample data points. One of them is the data used to evaluate the teacher's teaching process using students as the major evaluation body; the data used to evaluate the teacher's teaching process using students as the primary evaluation body is used as the evaluation model's input value; because it is crucial to the model's verification, the target expected output value is incorporated based on the evaluation of the teaching supervision group teachers' numerous lecture records. The model's target predicted output value is based on the score. During the study, the sample data with a high evaluation, a bad assessment, or data that was inconsistent with the facts were removed from the data set, leaving 6,178 sample data. Sample training data is critical in neural networks. Normalize the input samples to ensure that the data is within the specified range $[0,1]$, which is conducive to data processing and improves network efficiency. The normalization processing equation of the input sample is

$$
X=\frac{T-T_{\min }}{T_{\max }-T_{\min }} .
$$

4.3. Experimental Results. The improved gradient descent methodology is more effective than the standard gradient descent strategy in finding the adaptive learning rate and momentum term as the training function of a BP neural network. Assuming that the number of neurons in the hidden layer is 10 , the growth ratio and decline ratio of the adaptive learning rate are set as 1.2 and 0.7 , respectively, and the momentum term is set as 0.9. Input a training data set into the model for training in order to obtain stable network model, and then an input validation test data set, respectively,
TABLE 1: Comparison of the results of the experiment.

\begin{tabular}{lcc}
\hline Method & MSE & ACC \\
\hline Genetic algorithm & 48.32 & 0.7958 \\
BP & 46.99 & 0.8478 \\
Adaptive BP (ours) & 32.14 & 0.9120 \\
\hline
\end{tabular}

in two different algorithms as the training function, to contrast the performance of the model number of iterations and the training time, the MSE and model prediction accuracy comparison of the differences between predicted results and actual results. In this experiment, the average value obtained by running 10 times is the final result, and the result is shown in Table 1.The results show that when the adaptive learning rate and the gradient descent method with improved momentum term are used as the model's training functions, the learning rate of the model adapts adaptively and dynamically in response to changes in errors, ensuring that the model's convergence speed is improved during the training process, and the error between the model and the expected value of the target can be greatly reduced. It is also more effective than traditional methods in predicting accuracy and iteration times.

Figures 2 and 3 show that when the number of iterations grows, the MSE and prediction accuracy of the teaching evaluation model jump and fluctuate in the form of fluctuations, with the MSE increasing and then decreasing, but the ACC increasing all the while. Figure 4 shows that although the number of iterations and training duration of the model is not optimal when the number of neurons in the hidden layer is 30 , the difference is not significant when compared to the number of other neurons and can 


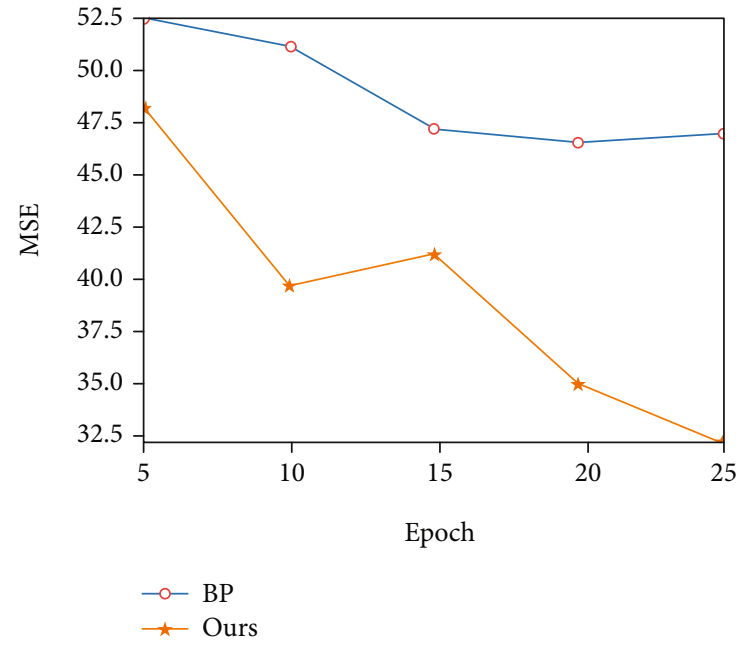

FIGURE 2: Change curve of convergence accuracy.

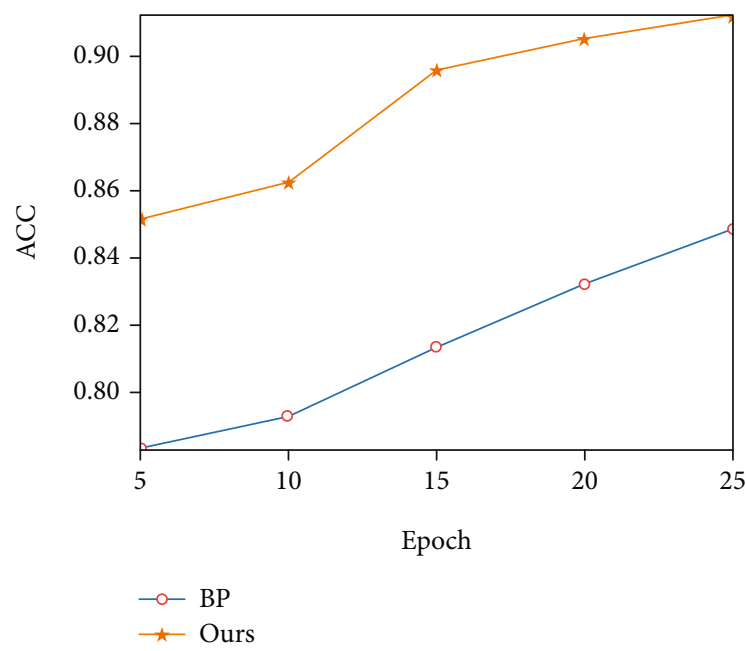

Figure 3: Change curve of ACC.

be tolerated; and at this time, the model of the MSE error and prediction accuracy rate are the best, which is more conducive to accurately evaluating teaching activities. As a result, based on the overall performance measures, the number of hidden layer neurons chosen is 30. Furthermore, this demonstrates that the method presented in this research can accurately assess the teaching quality of colleges and institutions.

4.4. Ablation Experiment. In order to further verify the effectiveness of the algorithm in this paper, we conducted ablation experiments to verify the influence of the BP network and multilayer perceptron (MLP) on the experimental results. The experimental results are shown in Table 2.

It can be seen from the experimental results in Table 2 that the performance of using MLP is far worse than that of using the BP network selected in this paper. Therefore, the MLP without optimized training is inferior to BP. Therefore, the proposed method uses the BP network.

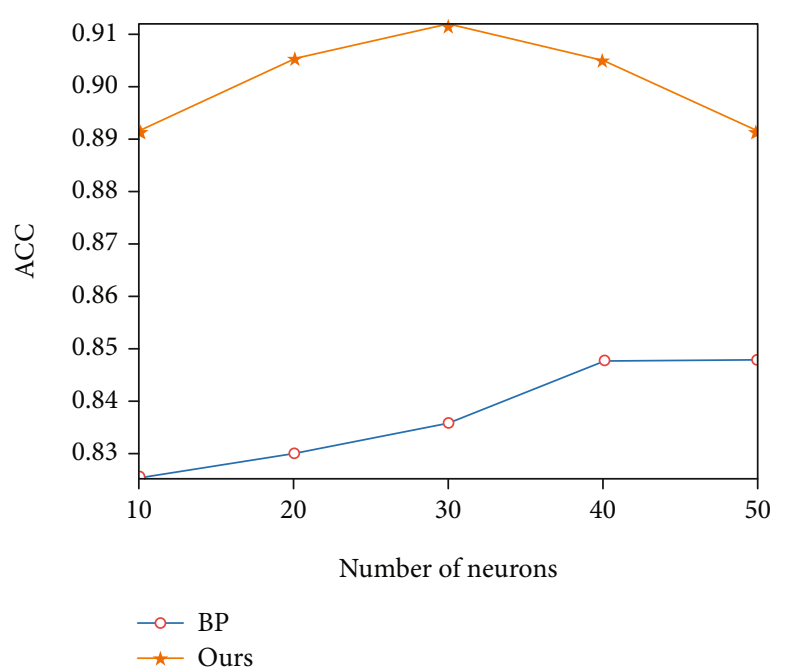

FIgURE 4: Change curve of neurons.

TABLE 2: Results of ablation experiments.

\begin{tabular}{lcc}
\hline Method & MSE & ACC \\
\hline Embed MLP & 49.51 & 0.8317 \\
Ours & 32.14 & 0.9120 \\
\hline
\end{tabular}

\section{Conclusion}

In this paper, a neural network model is used to solve complex nonlinear problems in the assessment of college teaching quality. It also performs an in-depth study on evaluating the quality of college instruction. The development of a new adaptive BP neural network model has been completed. To increase the model's convergence speed, optimize the network structure, and assure its stability, the model incorporates the adaptive vector and momentum of the modified gradient descent BP neural network. Then, to guarantee that the model completely evaluates educational activities, the new evaluation index was added to the traditional evaluation index. We also proved the effectiveness and superiority of the proposed model through experiments. In addition, the research in this paper is based on extensive data research and data cleaning in the early stage. The proposed algorithm will be limited to real-time processing in a complex environment.

\section{Data Availability}

The data used to support the findings of this study are included within the article.

\section{Conflicts of Interest}

All the authors do not have any possible conflicts of interest. 


\section{Acknowledgments}

This study was supported by the following projects: (1) Research Project of State Administration of Traditional Chinese Medicine with the Theme of "Extensive Learning, Deep Investigation, \& Detailed Implementation” (GY-15, Research \& Construction of TCM-Characterized Online General Courses) and (2) Science Popularization Project of Shanghai Municipal Science and Technology Commission (17dz2301000, Construction of TCM Science Popularization System Based on Cultural Inheritance \& Health Guidance).

\section{References}

[1] R. Lozano, M. Y. Merrill, K. Sammalisto, K. Ceulemans, and F. J. Lozano, "Connecting competences and pedagogical approaches for sustainable development in higher education: a literature review and framework proposal," Sustainability, vol. 9, no. 10, p. 1889, 2017.

[2] E. Abad-Segura and M. D. González-Zamar, "Sustainable economic development in higher education institutions: a global analysis within the SDGs framework," Journal of Cleaner Production, vol. 294, p. 126133, 2021.

[3] A. Syakur and Y. Sabat, "The effectiveness of coopertative learning (STAD and PBL type) on E-learning sustainable development in higher education," Journal of Development Research, vol. 4, no. 1, pp. 53-61, 2020.

[4] I. Molderez and E. Fonseca, "The efficacy of real-world experiences and service learning for fostering competences for sustainable development in higher education," Journal of Cleaner Production, vol. 172, pp. 4397-4410, 2018.

[5] H. A. Patrinos and N. Angrist, Global Dataset on Education Quality: A Review and Update (2000-2017), no. article 8592, 2018World Bank Policy Research Working Paper, 2018.

[6] M. Beerkens, "Evidence-based policy and higher education quality assurance: progress, pitfalls and promise," European Journal of Higher Education, vol. 8, no. 3, pp. 272-287, 2018.

[7] I. Degtjarjova, I. Lapina, and D. Freidenfelds, Student as Stakeholder: "Voice of Customer" in Higher Education Quality Development, Sumy State University, 2018.

[8] W. Zimmerman, B. Altman, B. Simunich, K. Shattuck, and B. Burch, "Evaluating online course quality: a study on implementation of course quality standards," Online Learning, vol. 24, no. 4, pp. 147-163, 2020.

[9] P. M. Bigatel and S. Edel-Malizia, "Using the "Indicators of Engaged Learning Online" framework to evaluate online course quality," TechTrends, vol. 62, no. 1, pp. 58-70, 2018.

[10] W. J. Van de Grift, S. Chun, R. Maulana, O. Lee, and M. Helms-Lorenz, "Measuring teaching quality and student engagement in South Korea and the Netherlands," School Effectiveness and School Improvement, vol. 28, no. 3, pp. 337349, 2017.

[11] E. Salman, "Teaching quality evaluation: online vs. manually, facts and myths," Journal of Information Technology Education: Innovations in Practice, vol. 16, no. 1, pp. 277-290, 2017.

[12] M. Bameni Moghadam and S. R. Rafiey, "Evaluation of teaching quality of faculty members in higher education institutions: a case study of faculty of economics at Allameh Tabataba'i University," Quarterly of Educational Measurement, vol. 8, no. 29, pp. 1-22, 2017.
[13] A. Debroy, A. Ingole, and A. Mudey, "Teachers' perceptions on student evaluation of teaching as a tool for faculty development and quality assurance in medical education," Journal of Education and Health Promotion, vol. 8, 2019.

[14] N. Huang, "Analysis and design of university teaching evaluation system based on JSP platform," International Journal of Education and Management Engineering, vol. 7, no. 3, pp. 43-50, 2017.

[15] M. Gao, W. Cai, and R. Liu, "AGTH-Net: attention-based graph convolution-guided third-order hourglass network for sports video classification," Journal of Healthcare Engineering, vol. 2021, Article ID 8517161, 10 pages, 2021.

[16] C. Yan, G. Pang, X. Bai et al., "Beyond triplet loss: person reidentification with fine-grained difference-aware pairwise loss," IEEE Transactions on Multimedia, p. 1, 2021.

[17] J. Zhang, J. Sun, J. Wang, and X. G. Yue, "Visual object tracking based on residual network and cascaded correlation filters," Journal of Ambient Intelligence and Humanized Computing, vol. 12, no. 8, pp. 8427-8440, 2021.

[18] Y. Tong, L. Yu, S. Li, J. Liu, H. Qin, and W. Li, "Polynomial fitting algorithm based on neural network," ASP Transactions on Pattern Recognition and Intelligent Systems, vol. 1, no. 1, pp. 32-39, 2021.

[19] X. Ning, Y. Wang, W. Tian, L. Liu, and W. Cai, "A biomimetic covering learning method based on principle of homology continuity," ASP Transactions on Pattern Recognition and Intelligent Systems, vol. 1, no. 1, pp. 9-16, 2021.

[20] C. Wang, X. Bai, X. Wang et al., "Self-supervised multiscale adversarial regression network for stereo disparity estimation," IEEE Transactions on Cybernetics, pp. 1-14, 2020.

[21] X. Bai, C. Yan, H. Yang, L. Bai, J. Zhou, and E. R. Hancock, "Adaptive hash retrieval with kernel based similarity," Pattern Recognition, vol. 75, pp. 136-148, 2018.

[22] L. Zhang, X. Wang, X. Dong, L. Sun, W. Cai, and X. Ning, "Finger vein image enhancement based on guided triGaussian filters," ASP Transactions on Pattern Recognition and Intelligent Systems, vol. 1, no. 1, pp. 17-23, 2021.

[23] A. R. A. Salih, "Peer evaluation of teaching or" fear" evaluation: in search of compatibility," Higher Education Studies, vol. 3, no. 2, pp. 102-114, 2013. 